\title{
ARTICLE Evidence for a sex-specific contribution of polygenic load for anorexia nervosa to body weight and prefrontal brain structure in nonclinical individuals
}

\author{
Elisabeth J. Leehr ${ }^{1}$, Nils Opel ${ }^{1}$, Janina Werner ${ }^{1}$, Ronny Redlich ${ }^{1}$, Jonathan Repple ${ }^{1}$, Dominik Grotegerd ${ }^{1}$, Katharina Dohm ${ }^{1}$, \\ Janik Goltermann (iD) ${ }^{1}$, Joscha Böhnlein ${ }^{1}$, Azmeraw T. Amare (D) $^{2}$, Lisa Sindermann ${ }^{1}$, Katharina Förster ${ }^{1}$, Susanne Meinert ${ }^{1}$, \\ Verena Enneking ${ }^{1}$, Maike Richter ${ }^{1}$, Tim Hahn ${ }^{1}$, Bernhard T. Baune ${ }^{1,3,4}$ and Udo Dannlowski ${ }^{1}$
}

\begin{abstract}
Genetic predisposition and brain structural abnormalities have been shown to be involved in the biological underpinnings of anorexia nervosa (AN). Prefrontal brain regions are suggested to contribute through behavioral inhibition mechanisms to body weight. However, it is unknown if and to which extent biological correlates for AN might be present in individuals without clinical AN symptomatology. We therefore investigated the contribution of polygenic load for AN on body weight and prefrontal brain structure in a sample of $n=380$ nonclinical individuals. A polygenic score (PGS) reflecting the individual genetic load for the trait of anorexia nervosa was calculated. Structural MRI data were acquired and preprocessed using the cortical parcellation stream of FreeSurfer. We observed a significant PGS $\times$ sex interaction effect on body mass index (BMI), which was driven by a negative correlation between PGS and BMI in female participants. Imaging analyses revealed significant interaction effects of sex $\times$ PGS on surface area of the lateral orbitofrontal cortex (OFC), the pars orbitalis (PO), the rostral middle frontal gyrus (RMF) and the pars triangularis (PT) of the left frontal cortex. The interaction effects were driven by positive correlations between PGS and prefrontal surface areas in female participants and negative correlations in male participants. We furthermore found sex-specific associations between BMI and left RMF surface area as well as between BMI and left PO and left RMF thickness. Our findings demonstrate a sexspecific association between polygenic load for $\mathrm{AN}, \mathrm{BMI}$, and prefrontal brain structure in nonclinical individuals. Hence, this study identifies structural abnormalities associated with polygenic load for AN and BMI in brain regions deeply involved in behavioral inhibition and impulse regulation as candidate brain regions for future research.
\end{abstract}

Neuropsychopharmacology (2019) 44:2212-2219; https://doi.org/10.1038/s41386-019-0461-1

\section{INTRODUCTION}

Anorexia nervosa (AN) is among the mental disorders with the highest mortality rates [1-3]. With standardized mortality rates of up to $5.86 \%$ [4], and a lifetime prevalence of $1 \%$ in women and $0.5 \%$ in men AN represents a major problem for mental health care [5]. Prevalence of AN in males might be underestimated [6, 7] and clinical presentation of men with AN differs compared to their female counterpart $[7,8]$.

Beside psychological (e.g., traits associated with perfectionism) and developmental (e.g., adverse life events) factors, biological factors are relevant in the pathogenesis of AN [1]. More specifically, recent neuroimaging studies have provided first evidence for AN specific brain structural correlates: in AN patients increased volume of the medial orbitofrontal cortex (OFC) and the insular cortex was repeatedly demonstrated and seems to persist even after recovery $[9,10]$.

Structural MRI findings are furthermore supplemented by fMRI findings showing deviations in the reward-circuitry, including the insula and striatum [9, 10]. Kaye et al. [11] postulated two brain circuits as relevant in AN: (a) a ventral (limbic) circuit associated with the identification of and reaction to significant emotional stimuli and (b) a dorsal (cognitive) circuit involved in cognitive control functions and regulation of emotions. Accordingly, there is evidence that brain areas involved in these circuits show structural abnormalities [12-16]. Prefrontal brain regions are supposed to be highly relevant in $\mathrm{AN}$ and alterations in prefrontal regions are suggested to predispose for the development of an AN through behavioral inhibition of food intake, which is, beside other possible regulations behaviors like excessive exercise, responsible for underweight in $A N[15,17,18]$. The finding that incidence of AN is peaking during puberty, a time when especially prefrontal brain regions are restructured furthermore adds to the relevance of prefrontal brain regions in AN [19-21].

With an estimated heritability between 48 and 74\% [22], genetic factors are thought to contribute to the development of AN. Recent genome-wide association studies (GWAS) have identified several genetic variants, including neurodevelopmental genes, that are associated with AN [23]. Based on GWAS findings, others

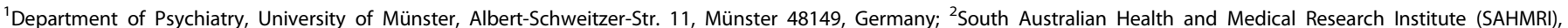

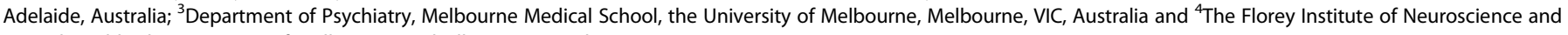
Mental Health, the University of Melbourne, Parkville, VIC, Australia

Correspondence: Udo Dannlowski (udo.dannlowski@uni-muenster.de)

These authors contributed equally: Elisabeth J. Leehr, Nils Opel

Received: 11 March 2019 Revised: 27 June 2019 Accepted: 1 July 2019

Published online: 8 July 2019 
Evidence for a sex-specific contribution of polygenic load for anorexia... EJ Leehr et al.

[24-27] and we [28-30] could recently show the benefits of applying polygenic scores (PGS) in neuroimaging studies on mental disorders and obesity suggesting that this approach could also be adopted for AN. PGS individually subsume genetic variants of small effects, identified in GWAS and therefore try to qualify cumulative effects of several genes. So far only few studies have applied PGS for AN [31-35]. In this regard, a recent publication of the "Enhancing Neurolmaging Genetics through Meta-Analysis Consortium" (ENIGMA) and the Eating Disorders Working Group of the "Psychiatric Genomics Consortium" (PGC-ED) has focused on the covariation of subcortical brain structure and genetic risk for AN [36] but failed to show significant associations. The authors of the aforementioned study suggested the following limitations of their work: lack of investigation of differential structural neuroimaging phenotypes like cortical thickness and surface, heterogeneity of the sample, and the lack of subgroup analysis. The latter notion particularly the analyses of potential sex-specific associations between genetic load, body mass index (BMI), and brain structure appears highly relevant considering that several previous studies found evidence for a sex-specific genetic contribution to BMI $[37,38]$ and to AN [39]. Further, there is evidence for sex-specific anthropometric and metabolic genetic determinants of body composition [40].

Therefore, with this study we aimed to investigate the contribution of polygenic load for AN to BMI and brain structure by taking into account potential limitations of previous research. In light of the aforementioned evidence from the literature, we hypothesized sex-specific contributions of polygenic load for AN on body weight and prefrontal brain structure in a middle-aged cohort of nonclinical individuals.

\section{METHODS}

Participants

This study comprised genetic and neuroimaging data from a total of 380 nonclinical adults as part of the Münster Neuroimage Cohort (MNC) acquired at the Department of Psychiatry, University of Münster (Table 1). Exclusion criteria comprise any history of neurological (e.g., concussion, stroke, tumor, and neuroinflammatory diseases) and medical (e.g., cancer, chronic inflammatory or autoimmune diseases, heart diseases, diabetes mellitus, and infections) conditions. All participants were ensured to be free from present or history of psychiatric disorders including Anorexia nervosa according to the Diagnostic and Statistical Manual of Mental Disorders criteria [DSM-IV, [41]] using the Structured Clinical Interview [SCID, [42]].

Information on body height and weight were collected based on self-report from each participant. Psychiatric symptomatology was assessed by means of the Beck Depression Inventory [43, 44], the State-Trait Anxiety Inventory [45], and the NEO-Five-FactorsInventory [46]. Intelligence quotient was ascertained with the multiple-choice vocabulary intelligence test [47] and BMI values were calculated as body weight in $\mathrm{kg} /(\text { body height in } \mathrm{m})^{2}$. All participants received a financial compensation. The study was approved by the local institutional review board (IRB), and written informed consent was obtained from all participants before study participation.

\section{sMRI methods}

Image acquisition. T1-weighted high-resolution anatomical images were acquired for all participants. Details of the imaging procedures for each cohort have been extensively described elsewhere (see Supplementary Methods) [29, 48, 49].

FreeSurfer. All structural images were preprocessed using the cortical parcellation stream of FreeSurfer (Version 5.3) with default parameters (https://surfer.nmr.mgh.harvard.edu/). Segmentation
Table 1. Sociodemographic sample characteristics

\begin{tabular}{|c|c|c|c|}
\hline & Mean (SD) & Min & Max \\
\hline \multicolumn{4}{|l|}{ Male $(n=184)$} \\
\hline Age & $38.28(11.04)$ & 21.00 & 59.00 \\
\hline BMI & $25.35(3.74)$ & 18.17 & 39.55 \\
\hline BDI & $1.76(2.53)$ & 0 & 14.00 \\
\hline STAI & $31.15(6.41)$ & 20.00 & 53.00 \\
\hline Verbal IQ & $116.04(12.94)$ & 92.00 & 145 \\
\hline Education (in years) & $15.28(2.30)$ & 9.00 & 21.00 \\
\hline \multicolumn{4}{|l|}{ Female $(\boldsymbol{n}=196)$} \\
\hline Age & 39.78 (11.50) & 18.00 & 59.00 \\
\hline BMI & $23.96(4.21)$ & 18.31 & 42.21 \\
\hline $\mathrm{BDI}$ & $2.24(3.15)$ & 0 & 26.00 \\
\hline STAI & $32.24(7.30)$ & 20.00 & 58.00 \\
\hline Verbal IQ & $115.81(12.28)$ & 92 & 145 \\
\hline Education (in years) & $15.04(2.5)$ & 9.00 & 24.00 \\
\hline \multicolumn{4}{|l|}{ Total $(n=380)$} \\
\hline Age & $39.06(11.29)$ & 18.00 & 59.00 \\
\hline BMI & $24.63(4.04)$ & 18.17 & 42.21 \\
\hline $\mathrm{BDI}$ & $2.01(2.87)$ & 0 & 26.00 \\
\hline STAI & $31.71(6.90)$ & 20.00 & 58.00 \\
\hline Verbal IQ & $115.81(12.28)$ & 92.00 & 145 \\
\hline Education (in years) & $15.16(2.32)$ & 9.00 & 24.00 \\
\hline
\end{tabular}

quality was assessed by visual inspection and furthermore statistically evaluated for outliers following a standardized protocol provided by the ENIGMA consortium (http://enigma.ini. usc.edu/protocols/imaging-protocols). Since cortical surface area scales with overall head size, regional specificity of cortical surface area alterations was ascertained by including total intracranial volume (ICV) as nuisance regressor in all analyses of cortical surface area.

\section{Genetic methods}

Genotyping in all participants was performed using previously published protocols (see Supplementary Methods) [29, 48]. Quality-controlled single nucleotide polymorphisms (SNPs) were clumped for linkage disequilibrium based on GWAS association $p$-value informed clumping using $r^{2}=0.1$ within a $250-\mathrm{kb}$ window to create a SNP-set in linkage equilibrium using PLINK software [50] run on Linux (plink -clump-p1 1 -clump-p2 1 -clump-r2 0.1 -clump-kb 250). A total of eight PGS for AN were generated and applied for this study using set of SNPs selected based on $p$-value thresholds ranging from 0.001 to 1.0 for each participant (see supplementary Table 1). The PGS were calculated as the sum of independent SNPs (genotype dosage from 0 to 2) of the reference allele in in the target samples weighted by GWAS effect sizes for the reference allele obtained from AN GWAS [23]. The PGS were computed using PLINK-1.9 [50]. Standardized PGS values based on $z$-transformation were used for all analyses. To adjust for genetic heterogeneity within our samples four multi-dimensional scaling (MDS) components resulting from the genetic population stratification analyses in PLINK were used. 


\section{Statistical analyses}

SPSS (IBM) version 25 was used for all statistical analyses. In order to address our hypotheses, the following analyses steps were carried out.

Polygenic score (PGS)-body mass index (BMI). First, in order to select the polygenic score that explained most variance in BMI, separate regression analyses were carried out revealing that PGS with the $p$-value threshold $p=0.001$ explained most variance in BMI $(B=-0.305, \beta=-0.075, t=-1.530, p=0.127)$. This PGS score was used for all subsequent analyses. (Results for associations between PGS and BMI for all calculated PGS can be found in the supplementary Table S1.)

In order to explore associations between the PGS and BMI, an analysis of covariance (ANCOVA) including age and MDS components as covariates of no interest, sex, PGS as well as the $\mathrm{PGS} \times$ sex interaction as predictors and $\mathrm{BMI}$ as dependent variable was carried out. To identify the direction of the observed association, bivariate correlation analyses between the PGS and $\mathrm{BMI}$ were carried out for each sex subgroup.

PGS-cortical surface. In line with our hypothesis, we first sought to investigate associations between PGS and prefrontal brain structure. To this end, we assessed associations between cortical surface measures of all 22 frontal cortical regions included in the FreeSurfer cortical processing stream based on the Desikan-Killiany atlas [51]. Associations were investigated by using a similar ANCOVA model as compared to analysis step (1) with sex and PGS as predictor variable and by including age and MDS components as nuisance regressors. ICV was included for analyses of cortical surface area. In line with the aforementioned analysis step main effects of PGS as well as interaction effects of PGS $\times$ sex were assessed. FDR correction for multiple comparisons using the Benjamini-Hochberg correction with a $q<0.05$ for a total of 22 tests was carried out. In addition, to assess potential associations between PGS and surface area outside the frontal cortex, exploratory analyses of associations with surface area across the entire cortex were carried out using similar models with all 68 cortical regions as dependent variables.

PGS-cortical thickness. The aforementioned analyses steps under (2) were repeated by including thickness instead of surface measures as dependent variables using an identical statistical model. As thickness does not scale with head size, ICV was not included as nuisance regressor in this analyses step.

Associations between BMI, PGS, and cortical brain structure. To investigate potential associations between BMI and prefrontal brain structure, similar linear models as applied in the aforementioned analyses steps (2) and (3) were conducted by including surface and thickness measures of all 22 frontal cortical regions as dependent variables including $\mathrm{BMI}$ as predictor and accounting for age, sex, and for analyses of surface measures for ICV. In addition, to further delineate the association between PGS, prefrontal brain structure, and BMI mediation analyses were applied.

\section{RESULTS}

Polygenic score (PGS)-body mass index (BMI)

We observed expected significant main effects of sex $\left(F_{(1,371)}=\right.$ $\left.14.402, p<0.001, \eta_{p}{ }^{2}=0.037\right)$, and age $\left(F_{(1,371)}=31.678, p<0.001\right.$, $\left.\eta_{p}{ }^{2}=0.079\right)$ on BMl. A trend for a main effect of PGS $\left(F_{(1,371)}=\right.$ $\left.2.825, p=0.094, n_{p}{ }^{2}=0.008\right)$ was qualified by a significant PGS $\times$ sex interaction effect on $\mathrm{BMI}\left(F_{(1,371)}=5.995, p=0.015, \eta_{p}{ }^{2}=\right.$ 0.016) (Fig. 1).

Bivariate correlations for sex subgroups revealed only for the female subsample a significant negative association of PGS and BMI $\left(r_{p}=-0.215, p=0.002\right)$ while no significant associations were found for the male subsample $\left(r_{p}<0.001, p=0.999\right)$.

No significant associations between PGS and further clinical or demographic variables occurred (see Supplementary results for details).

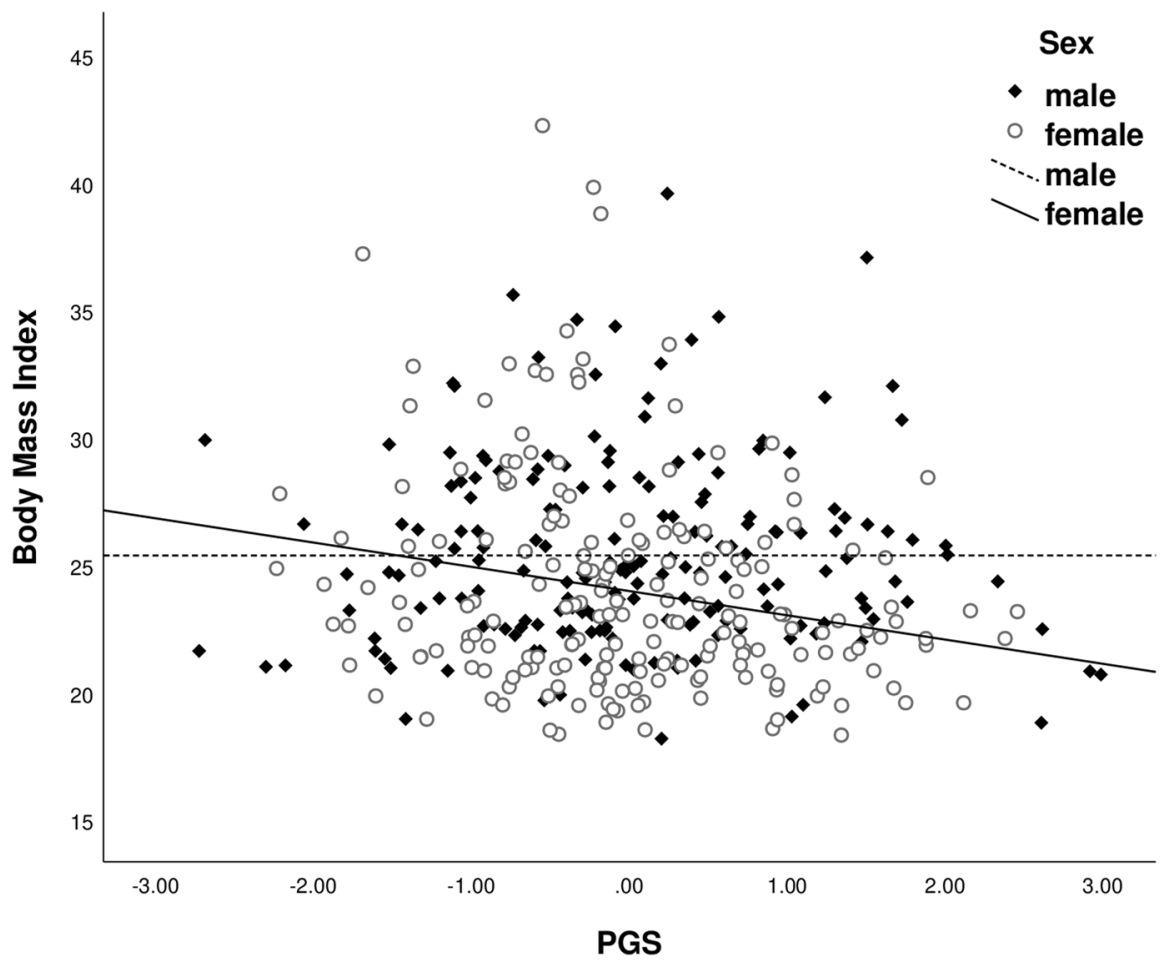

Fig. 1 Plot depicting the association between polygenic load scores (PGS) and BMI. PGS Polygenic load score, BMI Body mass index 

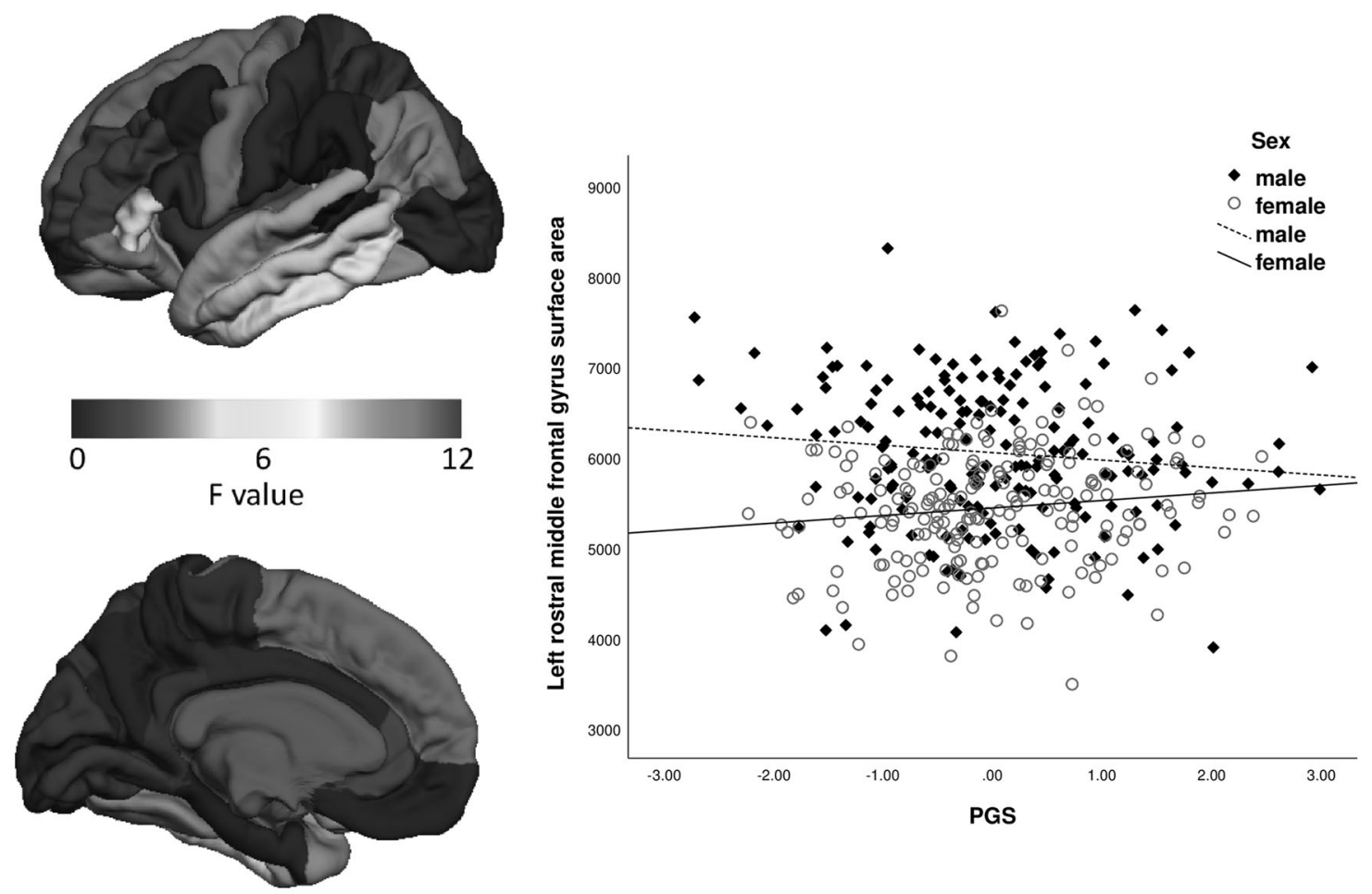

Fig. 2 Averaged $F$ value for all participants in cortical FreeSurfer regions projected onto the brain surface. Plot depicting the association between polygenic scores (PGS) and left rostral middle frontal gyrus surface area, PGS Polygenic score

PGS-cortical surface

We observed FDR corrected significant PGS $\times$ sex interaction effects on surface area of the left lateral orbitofrontal cortex $\left(F_{(1,369)}=\right.$ 9.791, $\left.p=0.002, \eta_{p}^{2}=0.026\right)$, the pars orbitalis of the left frontal cortex (PO: $\left.F_{(1,369)}=10.742, p=0.001, \eta_{p}{ }^{2}=0.028\right)$, the left rostral middle frontal gyrus (RMF: $F_{(1,369)}=12.000, p=0.001, \eta_{p}{ }^{2}=0.031$ ) (Fig. 2) and the left pars triangularis of the left frontal cortex (PT: $\left.F_{(1,369)}=8.163, p=0.005, \eta_{p}{ }^{2}=0.022\right)$. The observed interaction effects were driven by negative correlations between PGS and prefrontal surface area in male participants (OFC: $r_{p}=-0.159, p=$ 0.032; PO: $r_{p}=-0.207, p=0.005$; RMF: $r_{p}=-0.110, p=0.139$; PT: $\left.r_{p}=-0.179, p=0.015\right)$ and positive correlations in female participants (OFC: $r_{p}=0.080, p=0.267$; PO: $r_{p}=0.066, p=0.360$; RMF: $r_{p}=0.127, p=0.076$; PT: $\left.r_{p}=0.071, p=0.324\right)$. No FDR corrected significant main effect of PGS could be observed (Table 2).

At an exploratory threshold of $p<0.05$ uncorrected, a main effect of PGS on left frontal pole area emerged $\left(F_{(1,369)}=4.437, p=0.035\right.$, $\left.\eta_{p}{ }^{2}=0.012\right)$. According to the FDR corrected PGS $\times$ sex interaction effects on surface area of the left frontal cortex, sex $\times$ PGS interaction effect equivalents on surface area of the right frontal cortex for $\mathrm{PO}$ $\left(F_{(1,369)}=5.369, p=0.021, \eta_{p}{ }^{2}=0.014\right)$ and $\operatorname{RMF}\left(F_{(1,369)}=5.971\right.$, $\left.p=0.015, \eta_{p}{ }^{2}=0.016\right)$ could be observed at this exploratory threshold (see Table 2 for detailed overview). The significant interaction of PGS $\times$ sex on prefrontal surface area could be replicated in analyses in the subsample of normal-weight participants (BMI range 18.5-30) (see Supplementary results for details).

Additional analyses of cortical regions throughout the entire cortex revealed no further FDR corrected significant main effects of PGS nor PGS $\times$ sex interaction effects on any cortical region outside the frontal cortex.

PGS-cortical thickness

No significant main effect of PGS or interaction effect of PGS $\times$ sex on cortical thickness could be detected at the applied FDR corrected thresholds. At an exploratory threshold of $p<0.05$ uncorrected, an interaction effect of PGS $\times$ sex on thickness of the pars triangularis of the left frontal cortex $\left(F_{(1,369)}=6.138, p=0.014\right.$, $\eta_{p}{ }^{2}=0.016$ ) was evident (see supplementary Table S2). Additional analyses of cortical regions throughout the entire cortex revealed no FDR corrected significant main effects of PGS nor PGS $\times$ sex interaction effects on any cortical region outside the frontal cortex.

Associations between BMI, PGS, and cortical brain structure Analyses of potential main effects of $\mathrm{BMI}$ and $\mathrm{BMI} \times$ sex interaction effects on frontal cortical surface area yielded no significant main effect of BMI, while a BMI $\times$ sex interaction effect on left rostral middle frontal surface area was observed $(F(1,369)=8.789, p=$ $0.003, \eta_{p}{ }^{2}=0.023$ ) of comparable effect size compared to PGS $\times$ sex interaction effects observed in analyses step (2) in the same cortical region but however marginally failed to reach FDR significance (see supplementary Table S3). The interaction effect of $\mathrm{BMI} \times$ sex on frontal cortical surface area was driven by a more pronounced negative association between $\mathrm{BMI}$ and surface area in male compared to female participants (Fig. S1).

Analyses of main effects of BMI and BMI $\times$ sex interaction effects on frontal cortical thickness yielded several FDR corrected significant main and interaction effects with most pronounced interaction effects on thickness of the left pars opercularis and the left RMF (see supplementary Table S4). Again, interaction effects of $\mathrm{BMI} \times$ sex on frontal cortical thickness were driven by a more pronounced negative association between BMI and thickness in male compared to female participants (Figs. S2 and S3).

Additional mediation analyses revealed no significant indirect (mediation) effect of PGS on BMI through prefrontal surface area (see Supplementary results for details).

\section{DISCUSSION}

With this study we provide evidence for a sex-specific contribution of polygenic load for AN to BMI and prefrontal brain structure in nonclinical individuals. 
Our findings demonstrate a sex-specific negative association between polygenic load for AN and BMI in female participants, whereas this association was absent in males. This finding holds several important implications for future clinical and biological research. Since all participants of our study were ensured to be free from present or any history of eating disorders including AN, this observation might point to a sex-specific contribution of polygenic load for AN on BMI independent of AN diagnosis. This notion is furthermore supported by the fact that our analyses controlled for age and by the fact that the mean age of our sample (mean age $=39.06$, $S D=11.29$ ) was above the typical age range in which AN is diagnosed making it unlikely that findings were biased by individuals with a high probability of developing AN in the future. Instead, we demonstrate that the influence of polygenic load for AN on BMI does not seem to depend on AN diagnosis in nonclinical participants. Polygenic load for AN might play a relevant role for body weight regulation in nonclinical individuals. This goes in hand with the fact that apart from clinical manifest AN, symptoms like body and/or weight dissatisfaction are highly prevalent in the general population [52-55] and might result in co-occurrence with disordered eating behavior like extreme dieting [56-58]. The fact that the negative association between PGS and BMI was restricted to female participants is in accordance with the higher prevalence of $\mathrm{AN}$ as well as $\mathrm{AN}$ specific eating disorder related symptoms (weight dissatisfaction, dieting, weight control, and use of purging) in general among women compared to men [59]. Based on our observation, it appears likely that higher polygenic load for AN is associated with lower BMI in female individuals, which might be due to alterations in eating and weight regulation behavior. Still, alternative explanations for the association of PGS for AN and low BMI in nonclinical participants like differences in the tolerance or the function of starvation might be possible [60,61].

Interestingly, the assumption of an aberrant eating and weight regulation behavior appears to correspond to the observed imaging results. We found higher polygenic load for AN to be associated with higher left sided prefrontal surface area in the OFC, PO, RMF, and PT in female participants, with similar sex-specific effects in the right hemisphere for $\mathrm{PO}$ and RMF at an uncorrected level. These findings are of particular interest considering the crucial role of prefrontal cortical regions in the evaluation of stimuli valence and executive inhibitory control $[62,63]$. Moreover, prefrontal brain regions have frequently been demonstrated to be involved in eating disorders and weight regulation [64, 65]. The left orbitofrontal gyrus rectus gray matter volume has been shown to be increased in patients with acute $\mathrm{AN}$ as well as in recovered patients with $\mathrm{AN}$ and therefore alterations in this region might constitute a trait marker for AN [64]. Furthermore, alterations in inhibitory control are widely discussed as an explanation factor for altered eating behavior [66-68] and high inhibitory control abilities have been shown in patients with AN $[66,69,70]$. In addition, the frequent finding of decreased prefrontal gray matter volume in obesity, overeating and decreased impulse control, weight regulation, and reward sensitivity might further support this line of argumentation [71-73]. The fact, that we observed an influence of polygenic load for AN exclusively on cortical surface area corresponds to the fact that cortical surface area and thickness have been shown to be genetically independent [74-76].

Finally, we found a sex-specific association between BMI and left RMF surface area as well as between BMI and left PO and left RMF thickness. This association between BMI and lower prefrontal brain surface area and thickness was consistently more pronounced in men compared to women. We did not find evidence for a mediation of polygenic load for AN on BMI through prefrontal brain structure. This finding is in contrast with previous imaging genetic findings in obesity [29] and might 
be explained by the presence of further undiscovered variables (e.g., environmental factors) that are known to contribute to body weight.

The finding of sex-specific associations between both BMI and PGS with brain structure in prefrontal regions is supported by various studies including findings from different levels of biological abstraction: several studies show mutual as well as sex-specific associations of BMl and gray matter volume $[77,78]$. High BMI has been shown to affect the executive and reward system of the brain in elderly women [79], as well as neurodegeneration in women with Alzheimer's Dementia [80]. Some studies suggest common as well as sex-specific alterations regarding diffusion tensor imaging measures [81, 82]. A recent study found sex differences in metabolic brain age with lower metabolic brain age in women compared to men [83]. Sex-differences have also frequently been reported in gene expression patterns of high relevance for brain structural development $[84,85]$.

It appears important to note that the sex-specific effect of PGS for AN occurred only on prefrontal surface area, whereas the sexspecific effect of BMI showed up mainly in prefrontal thickness. This fact strengthens the argumentation of further underlying contributors to the association between body weight and brain structure beyond genetic influence.

The precise mechanisms underlying mutual sex-specific associations between polygenic risk for $\mathrm{AN}, \mathrm{BMI}$, and prefrontal structure currently remain unclear and should be targeted by future research. Future studies should take into account sexspecific gene expression that might help to unravel the complex picture of genes, BMl, and brain structure in body weight regulation.

Strengths of our work comprise the relatively large sample size in comparison with the previous studies on AN that enabled us to detect associations of small effect size as commonly observed in imaging genetic research. We accounted for multiple testing using the FDR correction for each analysis as it is standard in the field [e.g., [86]]. The inclusion of clinical, genetic, and neuroimaging data furthermore allows for abstraction on different levels. Since biological and imaging research on AN usually suffers from small sample sizes, a strong bias due to malnutrition and somatic as well as psychiatric comorbidities, the application of a PGS for AN and the successful application of the latter in imaging research in this study might prove as a useful approach for future research on AN. The application of a genetic score furthermore allowed us to disentangle between the temporal precedence of the predictor variable (PGS) and the studied outcome variables. Still, the cross-sectional design of our study represents a major limitation since temporal precedence between all other studied variables remains unclear and thus prevents us from drawing causal relationships. We furthermore cannot rule out that other variables (first and foremost environmental factors) have contributed to the detected associations. Moreover, the lack of any eating disorder related psychometric data apart from the SCID interview represents another limitation of the present study that must be acknowledged. As we included only nonclinical individuals, we cannot generalize results to individuals with diagnosed eating disorder.

To conclude our results suggest a sex-specific contribution of polygenic load on body weight and prefrontal brain structure, as well as a sex-specific association between BMI and prefrontal brain structure. These findings highlight a sex-specific role of AN related biological traits in nonclinical individuals. Future studies should aim to clarify to which extent similar sex-specific effects might be present in further domains of AN relevant neurobiology and to disentangle the complexity of these neurobiological mechanisms. Moreover, our results might point to the potential of sex-specific biologically informed prevention of disordered eating behavior in the general population that should be addressed in future research.

\section{FUNDING AND DISCLOSURES}

This work was funded by the German Research Foundation (DFG, grant FOR2107 DA1151/5-1 and DA1151/5-2 to UD; SFB-TRR58, Projects C09 and Z02 to UD) and the Interdisciplinary Center for Clinical Research (IZKF) of the medical faculty of Münster (grant Dan3/012/17 to UD grant SEED 11/18 to NO) and the Deanery of the Medical Faculty of the University of Münster. All authors state that they have no actual or potential conflict of interest to declare, including any financial, personal, or other relationships with other people or organizations within 3 years of beginning the submitted work that could influence or bias their work.

\section{ADDITIONAL INFORMATION}

Supplementary Information accompanies this paper at (https://doi.org/10.1038/ s41386-019-0461-1).

Publisher's note: Springer Nature remains neutral with regard to jurisdictional claims in published maps and institutional affiliations.

\section{REFERENCES}

1. Zipfel S, Giel KE, Bulik CM, Hay P, Schmidt U. Anorexia nervosa: aetiology, assessment, and treatment. Lancet Psychiatry. 2015;2:1099-111.

2. Zipfel S, Löwe B, Reas DL, Deter H-C, Herzog W. Long-term prognosis in anorexia nervosa: lessons from a 21-year follow-up study. Lancet. 2000;355:721-2.

3. Hoang U, Goldacre M, James A. Mortality following hospital discharge with a diagnosis of eating disorder: national record linkage study, England, 2001-2009. Int J Eat Disord. 2014;47:507-15.

4. Arcelus J, Mitchell AJ, Wales J, Nielsen S. Mortality rates in patients with anorexia nervosa and other eating disorders. A meta-analysis of 36 studies. Arch Gen Psychiatry. 2011;68:724-31.

5. Smink FR, van Hoeken D, Hoek HW. Epidemiology of eating disorders: incidence, prevalence and mortality rates. Curr Psychiatry Rep. 2012;14:406-14.

6. Limbers CA, Cohen LA, Gray BA. Eating disorders in adolescent and young adult males: prevalence, diagnosis, and treatment strategies. Adolesc Health Med Ther. 2018;9:111-6.

7. Murray SB, Nagata JM, Griffiths S, Calzo JP, Brown TA, Mitchison D, et al. The enigma of male eating disorders: acritical review and synthesis. Clin Psychol Rev. 2017:57:1-11.

8. Núñez-Navarro A, Agüera Z, Krug I, Jiménez-Murcia S, Sánchez I, Araguz N, et al. Do men with eating disorders differ from women in clinics, psychopathology and personality? Eur Eat Disord Rev. 2012;20:23-31.

9. Frank GK. Recent advances in neuroimaging to model eating disorder neurobiology. Curr Psychiatry Rep. 2015;17:559.

10. Frank GK. Advances from neuroimaging studies in eating disorders. CNS Spectr. 2015;20:391-400.

11. Kaye $W H$, Fudge $J$, Paulus $M$. New insights into symptoms and neurocircuit function of anorexia nervosa. Nat Rev Neurosci. 2009;10:573-84.

12. Friederich $\mathrm{H}-\mathrm{C}$, Walther $\mathrm{S}$, Bendszus $\mathrm{M}$, Biller $\mathrm{A}$, Thomann $\mathrm{P}$, Zeigermann $\mathrm{S}$, et al. Grey matter abnormalities within cortico-limbic-striatal circuits in acute and weight-restored anorexia nervosa patients. Neuroimage. 2012;59:1106-13.

13. Miles $A E$, Voineskos $A N$, French $L$, Kaplan AS. Subcortical volume and cortical surface architecture in women with acute and remitted anorexia nervosa: an exploratory neuroimaging study. J Psychiatr Res. 2018;102:179-85.

14. King JA, Frank GKW, Thompson PM, Ehrlich S. Structural neuroimaging of anorexia nervosa: future directions in the quest for mechanisms underlying dynamic alterations. Biol Psychiatry. 2018;83:224-34.

15. Frank GK. Altered brain reward circuits in eating disorders: chicken or egg? Curr Psychiatry Rep. 2013;15:396.

16. Van den Eynde F, Suda M, Broadbent H, Guillaume S, Van den Eynde M, Steiger $H$, et al. Structural magnetic resonance imaging in eating disorders: a systematic review of voxel-based morphometry studies. Eur Eat Disord Rev. 2012;20:94-105.

17. Volkow ND, Wang G-J, Tomasi D, Baler RD. The addictive dimensionality of obesity. Biol Psychiatry. 2013;73:811-8.

18. Miller E. The prefontral cortex and cognitive control. Nat Rev Neurosci. 2000;1:59-65.

19. Herpertz-Dahlmann B, Seitz J, Konrad K. Aetiology of anorexia nervosa: from a 'psychosomatic family model' to a neuropsychiatric disorder? Eur Arch Psychiatry Clin Neurosci. 2011;261:177-81.

20. Somerville LH, Casey B. Developmental neurobiology of cognitive control and motivational systems. Curr Opin Neurobiol. 2010;20:236-41. 
21. Fujisawa TX, Yatsuga C, Mabe H, Yamada E, Masuda M, Tomoda A. Anorexia nervosa during adolescence is associated with decreased gray matter volume in the inferior frontal gyrus. PLoS ONE. 2015;10:e0128548.

22. Yilmaz Z, Hardaway JA, Bulik CM. Genetics and epigenetics of eating disorders. Adv Genom Genet. 2015;5:131-50.

23. Boraska V, Franklin CS, Floyd JAB, Thornton LM, Huckins LM, Southam L, et al. A genome-wide association study of anorexia nervosa. Mol Psychiatry. 2014;19:1085-94.

24. Lancaster TM, Ihssen I, Brindley LM, Linden DE. Preliminary evidence for genetic overlap between body mass index and striatal reward response. Transl Psychiatry. 2018;8:19.

25. Wolf EJ, Miller DR, Logue MW, Sumner J, Stoop TB, Leritz EC, et al. Contributions of polygenic risk for obesity to PTSD-related metabolic syndrome and cortical thickness. Brain Behav Immun. 2017;65:328-36.

26. Andersen AM, Pietrzak RH, Kranzler HR, Ma L, Zhou H, Liu X, et al. Polygenic scores for major depressive disorder and risk of alcohol dependence. JAMA Psychiatry. 2017:74:1153.

27. Milaneschi Y, Lamers F, Peyrot WJ, Baune BT, Breen G, Dehghan A, et al. Genetic association of major depression with atypical features and obesity-related immunometabolic dysregulations. JAMA Psychiatry . 2017;74:1214.

28. Opel N, Redlich R, Repple J, Kaehler C, Grotegerd D, Dohm K, et al. Childhood maltreatment moderates the influence of genetic load for obesity on reward related brain structure and function in major depression. Psychoneuroendocrinology. 2019;100:18-26.

29. Opel N, Redlich R, Kaehler C, Grotegerd D, Dohm K, Heindel W, et al. Prefrontal gray matter volume mediates genetic risks for obesity. Mol Psychiatry. 2017;22:703-10.

30. Opel N, Amare AT, Redlich R, Repple J, Kaehler C, Grotegerd D, et al. Cortical surface area alterations shaped by genetic load for neuroticism. Mol Psychiatry. 2018. https://doi.org/10.1038/s41380-018-0236-9

31. Coleman JRI, Lester KJ, Keers R, Munafò MR, Breen G, Eley TC. Genome-wide association study of facial emotion recognition in children and association with polygenic risk for mental health disorders. Am J Med Genet B Neuropsychiatr Genet. 2017;174:701-11.

32. Abdellaoui A, Nivard MG, Hottenga J-J, Fedko I, Verweij KJH, Baselmans BML, et al. Predicting loneliness with polygenic scores of social, psychological and psychiatric traits. Genes Brain Behav. 2018;17:e12472.

33. Krapohl E, Patel H, Newhouse S, Curtis CJ, von Stumm S, Dale PS, et al. Multipolygenic score approach to trait prediction. Mol Psychiatry. 2018;23:1368-74.

34. Warrier V, Baron-Cohen S. Genetic contribution to 'theory of mind' in adolescence. Sci Rep. 2018;8:3465.

35. Socrates A, Bond T, Karhunen V, Auvinen J, Rietveld CA, Veijola J, et al. Polygenic risk scores applied to a single cohort reveal pleiotropy among hundreds of human phenotypes. Biorxiv.org. 2017.

36. Walton E, Hibar D, Yilmaz Z, Jahanshad N, Cheung J, Batury V-L et al. Exploration of shared genetic architecture between subcortical brain volumes and anorexia nervosa. Mol Neurobiol. 2018:1-11. https://doi.org/10.1007/s12035018-1439-4

37. Silventoinen K, Jelenkovic A, Sund R, Hur Y-M, Yokoyama Y, Honda C, et al. Genetic and environmental effects on body mass index from infancy to the onset of adulthood: an individual-based pooled analysis of 45 twin cohorts participating in the collaborative project of development of anthropometrical measures in Twins (CODATwins) study. Am J Clin Nutr. 2016;104:371-9.

38. Silventoinen K, Jelenkovic A, Sund R, Yokoyama Y, Hur Y-M, Cozen W, et al. Differences in genetic and environmental variation in adult BMI by sex, age, time period, and region: an individual-based pooled analysis of 40 twin cohorts. Am J Clin Nutr. 2017;106:457-66.

39. Baker JH, Maes HH, Lissner L, Aggen SH, Lichtenstein P, Kendler KS. Genetic risk factors for disordered eating in adolescent males and females. J Abnorm Psychol. 2009;118:576-86.

40. Hübel C, Gaspar HA, Coleman JRI, Finucane H, Purves KL, Hanscombe KB, et al. Genomics of body fat percentage may contribute to sex bias in anorexia nervosa. Am J Med Genet Part B Neuropsychiatr Genet. 2018. https://doi.org/10.1002/ ajmg.b.32709

41. American Psychiatric Association. Diagnostic and statistical manual of mental disorders. Washington, DC: American Psychiatric Association; 1994.

42. Wittchen H-U, Wunderlich U, Gruschwitz S, Zaudig M. Strukturiertes klinisches interview für DSM-IV. Goettingen: Hogrefe; 1997.

43. Beck AT, Steer RA. Beck depression inventory: manual. San Antonio: The Psychological Corporation, Harcourt Brace Jovanovich.; 1987.

44. Hautzinger M, Bailer M, Worall H, Keller F. Beck Depressions-Inventar (BDI). Testhandbuch. Bern: Hans Huber; 1994.

45. Spielberger CD, Gorsuch RL, Lushene R, Vagg PR, Jacobs GA. State-trait anxiety inventory, manual for the state-trait anxiety inventory. Palo Alto: Consulting Psychologist Press; 1970.
46. Costa PT, McCrae RR. Revised NEO personality inventory (NEOPI- RTM) and NEO five factor inventory (NEO-FFI): professional manual. Odessa, Florida: Psychological Assessment Resources; 1992.

47. Lehrl S. Mehrfachwahl-wortschatz-intelligenztest MWT-B. 4. überarb. Göttingen: Hogrefe; 1999.

48. Dannlowski U, Grabe HJ, Wittfeld K, Klaus J, Konrad C, Grotegerd D, et al. Multimodal imaging of a tescalcin (TESC)-regulating polymorphism (rs7294919)-specific effects on hippocampal gray matter structure. Mol Psychiatry. 2015;20:398-404.

49. Dannlowski U, Kugel H, Grotegerd D, Redlich R, Opel N, Dohm K, et al. Disadvantage of social sensitivity: interaction of oxytocin receptor genotype and child maltreatment on brain structure. Biol Psychiatry. 2016;80:398-405.

50. Chang CC, Chow CC, Tellier LC, Vattikuti S, Purcell SM, Lee JJ. Secondgeneration PLINK: rising to the challenge of larger and richer datasets. Gigascience. 2015;4:7.

51. Desikan RS, Ségonne F, Fischl B, Quinn BT, Dickerson BC, Blacker D, et al. An automated labeling system for subdividing the human cerebral cortex on MRI scans into gyral based regions of interest. Neuroimage. 2006:31:968-80.

52. Fiske L, Fallon EA, Blissmer B, Redding CA. Prevalence of body dissatisfaction among United States adults: review and recommendations for future research. Eat Behav. 2014;15:357-65.

53. Wyssen A, Bryjova J, Meyer AH, Munsch S. A model of disturbed eating behavior in men: the role of body dissatisfaction, emotion dysregulation and cognitive distortions. Psychiatry Res. 2016;246:9-15.

54. Bearman SK, Presnell K, Martinez E, Stice E. The skinny on body dissatisfaction: a longitudinal study of adolescent girls and boys. J Youth Adolesc. 2006;35:217-29.

55. Frederick DA, Peplau LA, Lever J. The swimsuit issue: correlates of body image in a sample of 52,677 heterosexual adults. Body Image. 2006;3:413-9.

56. Loth KA, MacLehose R, Bucchianeri M, Crow S, Neumark-Sztainer D. Predictors of dieting and disordered eating behaviors from adolescence to young adulthood. J Adolesc Heal. 2014;55:705-12.

57. Neumark-Sztainer D, Wall M, Larson NI, Eisenberg ME, Loth K. Dieting and disordered eating behaviors from adolescence to young adulthood: findings from a 10-year longitudinal study. J Am Diet Assoc. 2011;111:1004-11.

58. Carrard I, Kruseman M, Marques-Vidal P. Desire to lose weight, dietary intake and psychological correlates among middle-aged and older women. The CoLaus study. Prev Med (Balt). 2018;113:41-50.

59. Striegel-Moore RH, Rosselli F, Perrin N, DeBar L, Wilson GT, May A, et al. Gender difference in the prevalence of eating disorder symptoms. Int J Eat Disord. 2009;42:471-4.

60. Södersten P, Bergh C, Zandian M, loakimidis I. Homeostasis in anorexia nervosa. Front Neurosci. 2014;8:234.

61. Brockmeyer $T$, Holtforth MG, Bents $H$, Kämmerer A, Herzog W, Friederich H-C. Starvation and emotion regulation in anorexia nervosa. Compr Psychiatry. 2012;53:496-501.

62. Sinha R. Role of addiction and stress neurobiology on food intake and obesity. Biol Psychol. 2018;131:5-13.

63. Arnsten AFT. Stress signalling pathways that impair prefrontal cortex structure and function. Nat Rev Neurosci. 2009;10:410-22.

64. Frank GKW, Shott ME, Hagman JO, Mittal VA. Alterations in brain structures related to taste reward circuitry in ill and recovered anorexia nervosa and in bulimia nervosa. Am J Psychiatry. 2013;170:1152-60.

65. Voon V, Morris LS, Irvine MA, Ruck C, Worbe Y, Derbyshire K, et al. Risk-taking in disorders of natural and drug rewards: neural correlates and effects of probability, valence, and magnitude. Neuropsychopharmacology 2015:40:804-12.

66. Bartholdy S, Rennalls SJ, Jacques C, Danby H, Campbell IC, Schmidt U, et al. Proactive and reactive inhibitory control in eating disorders. Psychiatry Res. 2017;255:432-40.

67. Bartholdy S, Dalton B, O'Daly OG, Campbell IC, Schmidt U. A systematic review of the relationship between eating, weight and inhibitory control using the stop signal task. Neurosci Biobehav Rev. 2016;64:35-62.

68. Smith KE, Mason TB, Johnson JS, Lavender JM, Wonderlich SA. A systematic review of reviews of neurocognitive functioning in eating disorders: the state-ofthe-literature and future directions. Int J Eat Disord. 2018;51:798-821.

69. Steinglass JE, Figner B, Berkowitz S, Simpson HB, Weber EU, Walsh BT. Increased capacity to delay reward in anorexia nervosa. J Int Neuropsychol Soc. 2012;18:773-80.

70. Steward T, Menchon JM, Jiménez-Murcia S, Soriano-Mas C, Fernandez-Aranda F. Neural network alterations across eating disorders: a narrative review of $\mathrm{fMRI}$ studies. Curr Neuropharmacol. 2018;16:1150-63.

71. Pannacciulli N, Del Parigi A, Chen K, Le DSNT, Reiman EM, Tataranni PA. Brain abnormalities in human obesity: a voxel-based morphometric study. Neuroimage. 2006;31:1419-25.

72. Val-Laillet D, Aarts E, Weber B, Ferrari M, Quaresima V, Stoeckel LE, et al. Neuroimaging and neuromodulation approaches to study eating behavior 
and prevent and treat eating disorders and obesity. Neuroimage Clin. 2015;8:1-31.

73. Lu X, Zhong Y, Ma Z, Wu Y, Fox PT, Zhang N, et al. Structural imaging biomarkers for bipolar disorder: Meta-analyses of whole-brain voxel-based morphometry studies. Depress Anxiety. 2018. https://doi.org/10.1002/da.22866

74. Panizzon MS, Fennema-Notestine C, Eyler LT, Jernigan TL, Prom-Wormley E, Neale $M$, et al. Distinct genetic influences on cortical surface area and cortical thickness. Cereb Cortex. 2009;19:2728-35.

75. Hogstrom LJ, Westlye LT, Walhovd KB, Fjell AM. The structure of the cerebral cortex across adult life: age-related patterns of surface area, thickness, and gyrification. Cereb Cortex. 2013;23:2521-30.

76. Winkler AM, Kochunov P, Blangero J, Almasy L, Zilles K, Fox PT, et al. Cortical thickness or grey matter volume? The importance of selecting the phenotype for imaging genetics studies. Neuroimage . 2010;53:1135-46.

77. Taki Y, Kinomura S, Sato K, Inoue K, Goto R, Okada K, et al. Relationship between body mass index and gray matter volume in 1428 healthy individuals. Obes (Silver Spring). 2008;16:119-24.

78. Horstmann A, Busse FP, Mathar D, Müller K, Lepsien J, Schlögl H, et al. Obesityrelated differences between women and men in brain structure and goaldirected behavior. Front Hum Neurosci. 2011;5:58.

79. Sala A, Malpetti M, Ferrulli A, Gianolli L, Luzi L, Perani D. Effects of high BMI on synaptic function and metabolic connectivity in the brain-evidence of gender difference. Diabetes. 2018;67:363-OR.
80. Malpetti M, Sala A, Vanoli EG, Gianolli L, Luzi L, Perani D. Unfavourable gender effect of high body mass index on brain metabolism and connectivity. Sci Rep. 2018;8:12584.

81. Mueller K, Anwander A, Möller HE, Horstmann A, Lepsien J, Busse F, et al. Sexdependent influences of obesity on cerebral white matter investigated by diffusion-tensor imaging. PLoS ONE. 2011;6:e18544.

82. Gupta A, Mayer EA, Hamadani K, Bhatt R, Fling C, Alaverdyan M, et al. Sex differences in the influence of body mass index on anatomical architecture of brain networks. Int J Obes (Lond). 2017;41:1185-95.

83. Goyal MS, Blazey TM, Su Y, Couture LE, Durbin TJ, Bateman RJ, et al. Persistent metabolic youth in the aging female brain. Proc Natl Acad Sci USA. 2019;116:3251-5.

84. Yuan Y, Chen Y-PP, Boyd-Kirkup J, Khaitovich P, Somel M. Accelerated agingrelated transcriptome changes in the female prefrontal cortex. Aging Cell. 2012;11:894-901.

85. Berchtold NC, Cribbs DH, Coleman PD, Rogers J, Head E, Kim R, et al. Gene expression changes in the course of normal brain aging are sexually dimorphic. Proc Natl Acad Sci USA. 2008;105:15605-10.

86. Schmaal L, Hibar DP, Sämann PG, Hall GB, Baune BT, Jahanshad N et al. Cortical abnormalities in adults and adolescents with major depression based on brain scans from 20 cohorts worldwide in the ENIGMA major depressive disorder working group. Mol Psychiatry. 2017; 22:900-9. 